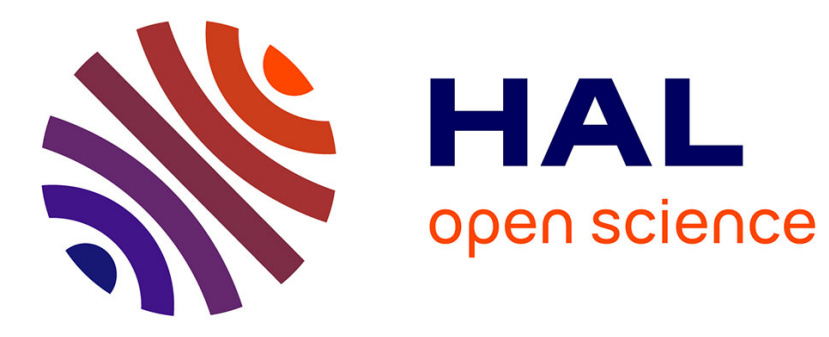

\title{
PDEs Level Sets on Weighted Graphs
}

Xavier Desquesnes, Abderrahim Elmoataz, Olivier Lezoray

\section{To cite this version:}

Xavier Desquesnes, Abderrahim Elmoataz, Olivier Lezoray. PDEs Level Sets on Weighted Graphs. International Conference on Image Processing (IEEE), Sep 2011, Bruxelles, Belgium. pp.3377-3380, 10.1109/ICIP.2011.6116433 . hal-00808939

\section{HAL Id: hal-00808939 \\ https://hal.science/hal-00808939}

Submitted on 8 Apr 2013

HAL is a multi-disciplinary open access archive for the deposit and dissemination of scientific research documents, whether they are published or not. The documents may come from teaching and research institutions in France or abroad, or from public or private research centers.
L'archive ouverte pluridisciplinaire HAL, est destinée au dépôt et à la diffusion de documents scientifiques de niveau recherche, publiés ou non, émanant des établissements d'enseignement et de recherche français ou étrangers, des laboratoires publics ou privés. 


\title{
PDES LEVEL SETS ON WEIGHTED GRAPHS
}

\author{
Xavier Desquesnes, Abderrahim Elmoataz, Olivier Lezoray \\ Université de Caen Basse Normandie \\ GREYC - UMR CNRS 6972 \\ 6.Bvd Marechal Juin, 14050 Caen, FRANCE
}

\begin{abstract}
In this paper we propose an adaptation of PDEs level sets over weighted graphs of arbitrary structure, based on PdEs and using a framework of discrete operators. A general PDEs level sets formulation is presented and an algorithm to solve such equation is described. Some transcriptions of well-known models under this formalism, as the mean-curvature-motion or active contours, are also provided. Then, we present several applications of our formalism, including image segmentation with active contours, using weighted graphs of arbitrary topologies.
\end{abstract}

Index Terms - PDEs, level sets, graphs, eikonal equation, front propagation.

\section{INTRODUCTION}

The partial differential equations (PDEs) provide a major mathematical tool in image processing, and are involved in the resolution of many problems such as image segmentation, object detection, image restoration or denoising, and so on. In this paper, we focus on the geometric PDEs for front propagation and their implementation by level set methods. The level set formulation to describe the curve evolution has been introduced by Osher-Sethian [1]. It provides well-known advantages such as treating self-intersections or topological changes and can be easily extended to $\mathbb{R}^{\mathrm{d}}$ with $d \geqslant 1$. Given a parametrized curve $\Gamma:[0,1] \rightarrow \Omega$, evolving on a domain $\Omega$ due to the effect of a scalar field $\mathcal{F}: \Omega \rightarrow \mathbb{R}$. The level set method aims to find a function $\phi(x, t)$ such that at each time $t$ the evolving curve $\Gamma_{t}$ can be provided by the 0-level set of $\phi$. In other words $\Gamma_{t}=\{x \mid \phi(x, t)=0\}$ and the curve evolution can be done solving

$$
\frac{\partial \phi}{\partial t}=\mathcal{F}|\nabla \phi|
$$

with initial condition $\phi(x, 0)=\phi_{0}(x)$, the initial embedding of $\Gamma$. A well known example of geometric PDEs in image processing is the mean curvature motion (MCM), $\frac{\partial \phi}{\partial t}=|\nabla \phi| \nabla \cdot\left(\frac{\nabla \phi}{|\nabla \phi|}\right)$, which found application in image denoising. One can also quote the active contours models summarized by $\frac{\partial \phi}{\partial t}=[\alpha \nabla \cdot(g(|\nabla \phi|) \nabla \phi)+\beta \mathcal{F}]|\nabla \phi|$, where $g$ is an edge detection function ( $g$ satisfies $g(x) \rightarrow 0$ when $x \rightarrow \infty), \alpha$ and $\beta$ are application dependent. Many variations of active contours models can be found in literature. One can remark that when $\mathcal{F}$ is positive or negative, equation (1) describes respectively morphological dilation and erosion process.

The level set approach to perform a front propagation is popular and literature is rich of efficient resolution methods. See [2] and references therein. Most of applications of these PDE based models focus either on image processing on Euclidean models, or on structured meshes. However, many other interesting applications involve data defined in more topologically complex domains: data defined on manifolds or irregularly shaped domains, data defined on network-like structures, or data defined as high dimensional point clouds such as collections of features vectors.

Thus, the objective of this paper is to extend this PDE based front propagation concept to graphs of arbitrary topology. Such a formulation enables the processing of a huge variety of discrete data that can be represented by a weighted graph, with applications in data clustering or image segmentation. Then, we propose to exploit the framework of PdEs on weighted graphs [3], to overcome the difficulties inherent in the geometric nature of the PDEs and propose a general formulation of geometric PDEs on graphs. Thus, we use a level set function $\phi: V \rightarrow\{-1,1\}$ as an implicit representation of the front and the evolution equation is given by

$$
\frac{\partial \phi}{\partial t}=\mathcal{F}\left|\nabla_{w} \phi\right|=\max (\mathcal{F}, 0)\left|\nabla_{w}^{-} \phi\right|+\min (\mathcal{F}, 0)\left|\nabla_{w}^{+} \phi\right|,
$$

where $\left|\nabla_{w}^{-} \phi\right|$ and $\left|\nabla_{w}^{+} \phi\right|$ are discrete versions of the gradient on graphs, which are defined in the sequel.

\section{PARTIAL DIFFERENCE EQUATIONS ON WEIGHTED GRAPHS}

We begin by briefly reviewing some basic definitions and operators on weighted graphs. 
Notations and Definitions. We assume that any discrete domain can be modeled by a weighted graph. Let $G=$ $(V, E, w)$ be a weighted graph composed of two finite sets: $V=\left\{u_{1}, \ldots u_{n}\right\}$ of $n$ vertices and $E \subset V \times V$ a set of weighted edges. An edge $(u, v) \in E$ connects two adjacent vertices $u$ and $v$. The weight $w_{u v}$ of an edge $(u, v)$ can be defined by a function $w: V \times V \rightarrow \mathbb{R}^{+}$if $(u, v) \in E$, and $w_{u v}=0$ otherwise. We denote by $N(u)$ the neighborhood of a vertex $u$, i.e. the subset of vertices that share an edge with $u$. In this paper, graphs are assumed to be connected, undirected and with no self loops.

Let $f: V \rightarrow \mathbb{R}$ be a discrete real-valued function that assigns a real value $f(u)$ to each vertex $u \in V$. We denote by $\mathcal{H}(V)$ the Hilbert space of such functions defined on $V$.

Operators on Weighted Graphs. For better comprehension of the next Section, we now quickly recall some operators on weighted graphs as they are defined in [3]. Considering a weighted graph $G=(V, E, w)$ and a function $f \in \mathcal{H}(V)$, the weighted discrete partial derivative operator of $f$ is

$$
\left(\partial_{v} f\right)(u)=\sqrt{w_{u v}}(f(v)-f(u)) .
$$

Based on this definition, two weighted directional difference operators are defined. The weighted directional external and internal difference operators are respectively :

$$
\begin{aligned}
& \partial_{v}^{+} f(u)=\sqrt{w_{u v}}(f(v)-f(u))^{+} \text {and } \\
& \partial_{v}^{-} f(u)=-\sqrt{w_{u v}}(f(v)-f(u))^{-}
\end{aligned}
$$

with $(x)^{+}=\max (0, x)$ and $(0)^{-}=\min (0, x)$.

The weighted gradient of a function $f \in \mathcal{H}(V)$ at vertex $u$ is the vector of all edge directional derivatives :

$$
\left(\nabla_{w} f\right)(u)=\left(\partial_{v} f(u)\right)_{(u, v) \in E}^{T}
$$

And the weighted morphological external and internal gradient $\left(\nabla_{w}^{+} f\right)(u)$ and $\left(\nabla_{w}^{-} f\right)(u)$ are :

$$
\left(\nabla_{w}^{ \pm} f\right)(u)=\left(\left(\partial_{v}^{ \pm} f\right)(u)\right)_{(u, v) \in E}^{T}
$$

Finally, the graph curvature [4] is given by:

$$
\kappa_{w}(u)=\sum_{v \sim u} \gamma_{w}(u, v)(f(u)-f(v)),
$$

with $\gamma_{w}(u, v)=w_{u v}\left(\left|\nabla_{w} f(v)\right|^{-1}+\left|\nabla_{w} f(u)\right|^{-1}\right)$.

PdE based Morphology Let us consider $\mathcal{A}$, a subset of $V$. The outer and inner boundary sets of $\mathcal{A}$ are respectively denoted $\partial^{+} \mathcal{A}$ and $\partial^{-} \mathcal{A}$. With $\partial^{+} \mathcal{A}=\left\{u \in \mathcal{A}^{c} \mid \exists v \in \mathcal{A}, v \sim\right.$ $u\}$ and $\partial^{-} \mathcal{A}=\left\{u \in \mathcal{A} \mid \exists v \in \mathcal{A}^{c}, v \sim u\right\}$ where $\mathcal{A}^{c}$ is the complement of $\mathcal{A}$. It was recently shown [3] that for any level set $f^{t}=\chi(f-t)$ of a function $f$, at a vertex $u \in V$, the $\mathcal{L}_{p}$ norm of the gradient $\left(\nabla_{w} f\right)(u)$ can be decomposed as

$$
\left\|\left(\nabla_{w} f^{t}\right)(u)\right\|_{p}^{p}=\left\|\left(\nabla_{w}^{+} f^{t}\right)(u)\right\|_{p}^{p}+\left\|\left(\nabla_{w}^{-} f^{t}\right)(u)\right\|_{p}^{p} .
$$

Based on these formulations, the discrete analogue of the continuous PDEs based dilation and erosion formulation can be defined as shown in the sequel.

Continuous scale morphology [5] defines flat dilation $\delta$ : $\mathbb{R}^{\mathrm{n}} \rightarrow \mathbb{R}^{\mathrm{n}}$ and erosion $\epsilon: \mathbb{R}^{\mathrm{n}} \rightarrow \mathbb{R}^{\mathrm{n}}$ of a function $f^{0}: \mathbb{R}^{\mathrm{m}} \rightarrow \mathbb{R}$ by structuring sets $B=\left\{x:\left\|x_{p}\right\| \leqslant 1\right\}$, with the general PdEs

$$
\frac{\partial \delta(f)}{\partial t}=\partial_{t} f=+\|\nabla f\|_{p} \text { and } \frac{\partial \epsilon(f)}{\partial t}=\partial_{t} f=-\|\nabla f\|_{p}
$$

Recently, graph based versions of these PdEs have been proposed in [3] as

$$
\frac{\partial \delta(f(u))}{\partial t}=\partial_{t} f(u)=+\left\|\left(\nabla_{w}^{+} f\right)(u)\right\|_{p}
$$

and

$$
\frac{\partial \epsilon(f(u))}{\partial t}=\partial_{t} f(u)=-\left\|\left(\nabla_{w}^{-} f\right)(u)\right\|_{p}
$$

Remarks. In the sequel we will always consider the $\mathcal{L}_{2}$ norm.

\section{TRANSCRIPTION OF GEOMETRIC PDES ON GRAPHS}

In this section, we present our transcription of geometric PDEs on weighted graphs of arbitrary topology and a new algorithm to describe the propagation of a front on such graphs.

Let $G(V, E, w)$ be a weighted graph. A front evolving on $G$ is defined as a subset $\Omega_{0} \subset V$, and is implicitly represented at initial time by a level set function $\phi_{0}=\mathcal{U}_{0}=\chi_{\Omega_{O}}-\chi_{\Omega_{0}^{c}}$, where $\chi: V \rightarrow\{0,1\}$ is the indicator function and $\Omega_{0}^{c}$ is the complement of $\Omega_{0}$. In other words $\phi_{0}$ equals 1 in $\Omega_{0}$ and -1 on its complementary.

Then, from the general equation (1) transposed on graphs, the front propagation can be described by

$$
\left\{\begin{array}{l}
\frac{\partial \phi}{\partial t}(u)=\mathcal{F}(u)\left\|\left(\nabla_{w} \phi\right)(u)\right\| \\
\phi_{0}(u)=\mathcal{U}_{0}
\end{array}\right.
$$

with $\mathcal{F} \in \mathcal{H}(V)$, and $w: V \times V \rightarrow \mathbb{R}^{+}$is the weight function. Based on the previous definition of discrete dilation and erosion on graphs (9), (10), the front propagation can be expressed as a morphological process with the following sum of dilation and erosion.

$$
\left\{\begin{array}{l}
\frac{\partial \phi}{\partial t}(u)=(\mathcal{F})^{+}\left\|\left(\nabla_{w}^{+} \phi\right)(u)\right\|+(\mathcal{F})^{-}\left\|\left(\nabla_{w}^{-} \phi\right)(u)\right\| \\
\phi_{0}(u)=\mathcal{U}_{0}
\end{array}\right.
$$

To solve this dilation and erosion process, on the contrary to the PDEs case, no spatial discretization is needed thanks to derivatives directly expressed in a discrete form. Then, the general iterative scheme to compute $\phi$ at time $t+1$ for all 
$u \in V$ is given by:

$$
\begin{array}{r}
\phi^{t+1}(u)=\phi^{t}(u)+\Delta t\left[(\mathcal{F}(u))^{+}\left\|\left(\nabla_{w}^{+} \phi^{t}\right)(u)\right\|+\right. \\
\left.(\mathcal{F}(u))^{-}\left\|\left(\nabla_{w}^{-} \phi^{t}\right)(u)\right\|\right]
\end{array}
$$

At each time $t+1$, the new value at a vertex $u$ only depends on its value at time $t$ and the existing values in its neighborhood. This equation can be split in two independent equations, in function of the sign of $\mathcal{F}$ :

$$
\phi^{t+1}(u)= \begin{cases}\phi^{t}(u)+\Delta t \mathcal{F}(u)\left\|\left(\nabla_{w}^{+} \phi^{t}\right)(u)\right\| & , \mathcal{F}(u)>0 \\ \phi^{t}(u)+\Delta t \mathcal{F}(u)\left\|\left(\nabla_{w}^{-} \phi^{t}\right)(u)\right\| & , \mathcal{F}(u)<0,\end{cases}
$$

Such decomposition of the process in two independent equations for erosion and dilation processes enhances the computation of the solution because one only has to compute one morphological gradient at each iteration, for a given vertex. Moreover, one can remark that at initialization both two gradients are zero everywhere, except for vertices which lies in the inner and outer boundaries of $\Omega_{0}$. Then, the set of vertices to be updated at each iteration can be restricted to two inner and outer narrow bands, initialized respectively with $\partial^{-} \Omega_{0}$ and $\partial^{+} \Omega_{0}$ and updated over time with neighbors of vertices already in. The narrow bands growth follows the fronts evolution and to avoid them to become too large, the narrow bands are reinitialized periodically. Thus, each $\tau$ iterations, which correspond to a step $k$, the front is given by the set $\Omega_{k}=\left\{u \mid \phi^{k \tau}(u)>0\right\}$ and the associated level set function is also reinitialized as $\phi_{k}(u)=\mathcal{U}_{k}=\chi_{\Omega_{k}}(u)-\chi_{\Omega_{k}^{c}}(u)$. Then, the inner and outer narrow bands are respectively reinitialized as $\partial^{-} \Omega_{k}$ and $\partial^{+} \Omega_{k}$.

The entire process to perform the front evolution is summarized in Algo. 1 and some examples will be provided in the sequel. The condition to stop the algorithm is application dependent.

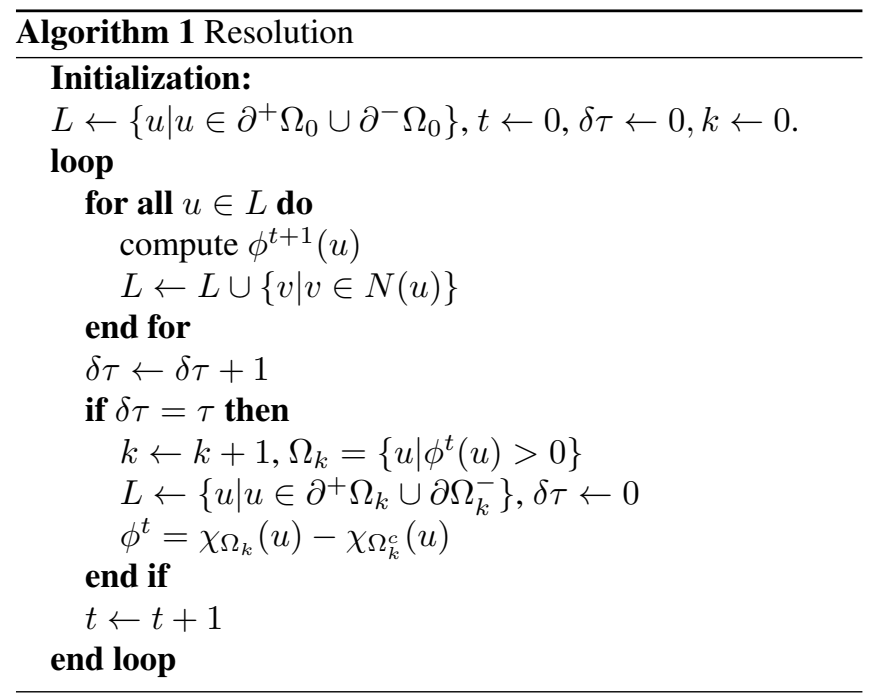

Eikonal Equation Using PDEs, and in the case where $\mathcal{F}$ is defined non-negative on the whole domain $\Omega$, the relation between the level set formulation (1) and the well-known eikonal equation $(\mathcal{F}(x)\|\nabla T(x)\|=1)$ stems from the following change of variable : $\phi(x, t)=t-T(x)$ (where $T(x)$ is the arrival time of the curve at a point $x$ ).

Using previous definitions of morphological evolution equations, one can formulate the same relation and obtain a PdEsbased version of the eikonal equation, defined on weighted graphs of arbitrary topology. Let $G(V, E, w)$ be a weighted graph (with functions $T$ and $\mathcal{F}$ defined on $\mathcal{H}(V)$ ). Because $\mathcal{F}$ is defined non-negative, the evolution process described by equation (12) can be seen as a dilation process and the evolution equation rewritten as

$$
\frac{\partial \phi}{\partial t}(u)=\mathcal{F}\left\|\left(\nabla_{w}^{+} \phi\right)(u)\right\| .
$$

With a similar change of variable $\phi(u)=t-T(u)$, we have $\frac{\partial \phi}{\partial t}(u)=\mathcal{F}\left\|\left(\nabla_{w}^{+}(t-T)\right)(u)\right\|=\mathcal{F}\left\|\left(\nabla_{w}^{-} T\right)(u)\right\|$, and then, and then,

$$
\mathcal{F}\left\|\left(\nabla_{w}^{-} T\right)(u)\right\|=1
$$

Numerical schemes and algorithms to solve such equation were provided in [6].

\section{EXAMPLES}

Mean Curvature Motion From the general morphological curve evolution equation (13), the mean curvature motion equation can be written as follows, where the speed $\mathcal{F}$ is given by the opposite of the graph curvature (??) :

$$
\frac{\partial \phi}{\partial t}(u)= \begin{cases}\left|\kappa_{w}^{\phi}(u)\right|\left\|\left(\nabla_{w}^{+} \phi\right)(u)\right\|, & \kappa_{w}^{\phi}(u)<0 \\ -\left|\kappa_{w}^{\phi}(u)\right|\left\|\left(\nabla_{w}^{-} \phi\right)(u)\right\|, & \kappa_{w}^{\phi}(u)>0\end{cases}
$$

where $\kappa_{w}^{\phi}$ is the graph curvature. Figure 1 presents an example of front regularization on an arbitrary graph, using the mean curvature motion.

Active Contours The following example will illustrate graph-based versions of eikonal equation and active contours, with our formulation of PDEs level sets on graphs, in a unique application. For this example, we adapt the well-known Chan $\&$ Vese active contour model [7] on weighted graphs. Using equation (13), the speed $\mathcal{F}$ is then defined as

$$
\mathcal{F}(u, t)=\beta \mathcal{K}_{w}(u)+\alpha\left(\left(u-\mu_{1}(t)\right)^{2}-\left(u-\mu_{2}(t)\right)^{2}\right)
$$

where $\mathcal{K}_{w}$ is a term of curvature, $\mu_{1}$ and $\mu_{2}$ are the mean outside the curve, respectively inside. $\alpha$ and $\beta$ are application dependent. The active contour evolution is performed on a reduced version of the initial image, a region adjacency graph 

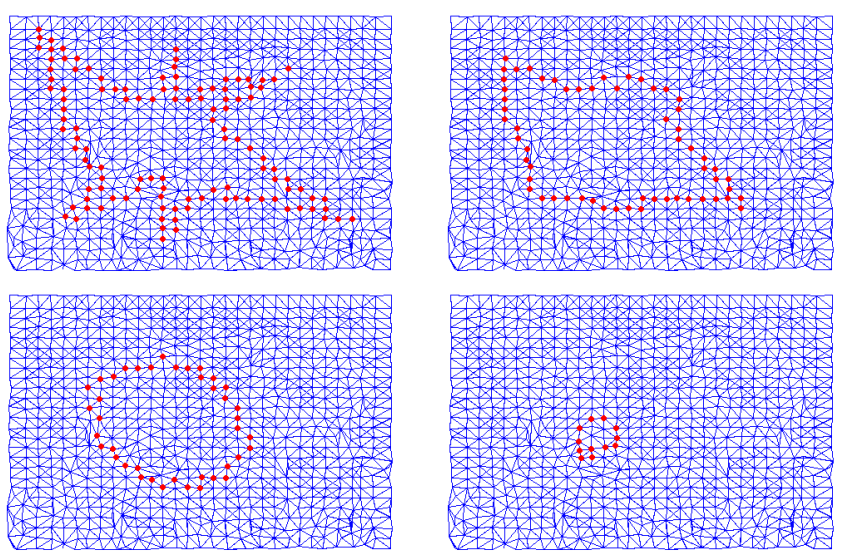

Fig. 1. An illustration of mean curvature motion on an arbitrary graph. The graph is weighted by a constant weight function $(w=1)$. Top-left image shows the initial front. Other images show the regularization of the front at different steps.
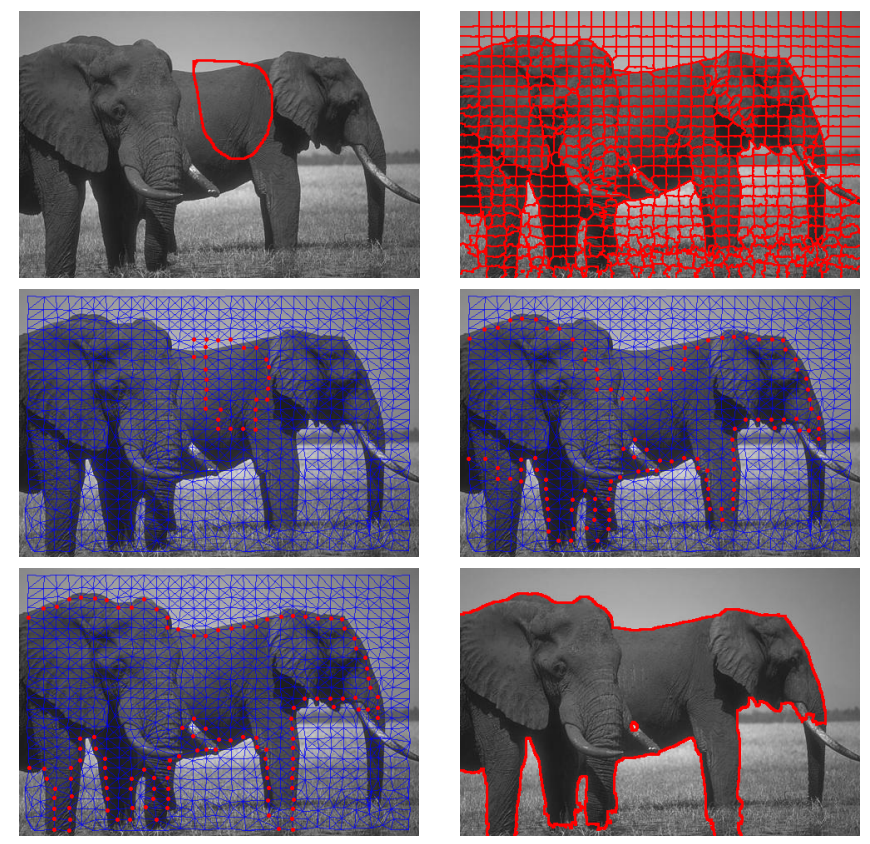

Fig. 2. An illustration of PDEs level set on graphs. The initial image is decomposed in regions using the eikonal equation, and a weighted RAG is built from the resulting region map. First row shows the initial image with superimposed initial front location and the region map. Second row shows the evolution of the active contour (red dots) on the graph (presented in blue) using PDEs based active contours. Third row presents the final contour on the graph, and its transposition on initial image. See text for details
(RAG), obtained from a superpixel decomposition. Such decomposition is performed using a regular-grid of seeds, which are dilated on the image 4-adjacency-grid graph using the PdEs based version of the eikonal equation (16). The resulting region map is then transformed in a second graph (the RAG) where each node is associated with a superpixel and edges represent the adjacency between superpixels. The two weight functions are computed from pixels intensity, respectively regions mean intensity. A detailed description of the method can be found in [6]. One can remark that due to its construction the second graph is irregular. Then, the active contour evolution is performed on this second graph, using our formulation of active contours on weighted graphs and Algo. 1. Finally, the contour at convergence of the algorithm is transposed from the graph to the region map and then to the original image. Figure 2 presents each steps of the entire process.

\section{CONCLUSION}

In this paper, we have presented an adaptation of PDEs level sets over weighted graphs, based on PdEs and using a framework of discrete operators. An algorithm to solve the proposed equation was also provided. Experimental results have shown the potentiality of the proposed formulation of PDEs level sets and its adaptivity to graphs of arbitrary topology.

\section{REFERENCES}

[1] J. A. Sethian, Level Set Methods and Fast Marching Methods, Cambridge University Press, 1999.

[2] T. F. Chan and J. Shen, Image Processing and Analysis, SIAM, 2005.

[3] V.-T. Ta, A. Elmoataz, and O. Lézoray, "Partial difference equations over graphs: Morphological processing of arbitrary discrete data," in Proceedings of ECCV, 2008, vol. LNCS 5304, pp. 668-680.

[4] A. Elmoataz, O. Lézoray, and S. Bougleux, "Nonlocal discrete regularization on weighted graphs: a framework for image and manifold processing.", IEEE Trans Image Process, vol. 17, no. 7, pp. 1047-60, 2008.

[5] R. W. Brockett and P. Maragos, "Evolution equations for continuous-scale morphology," in Proceedings of ICASSP, 1992, vol. 3, pp. 125-128.

[6] X. Desquesnes, A. Elmoataz, O. Lézoray, and V.-T. Ta, "Efficient algorithms for image and high dimensional data processing using eikonal equation on graphs," in Proceedings of ISVC, 2010, vol. 2, pp. 647-658.

[7] T. F. Chan and L. A. Vese, "Active contours without edges," IEEE Trans. on Image Processing, vol. 10, no. 2, pp. 266-277, Feb. 2001. 